\title{
An automated nucleic acid detection platform using digital microfluidics with an optimized Cas12a system
}

\author{
Zhen Sun $^{1 \dagger}$, Kang-Feng Lin ${ }^{1 \dagger}$, Ze-Hang Zhao ${ }^{1 \dagger}$, Yang Wang ${ }^{2}$, Xin-Xin Hong ${ }^{1}$, Jian-Guang Guo ${ }^{1}$, \\ Qing-Yu Ruan, Lian-Yu Lu ${ }^{2}$, Xiao Li ${ }^{1}$, Rui Zhang ${ }^{3 *}$, Chao-Yong Yang ${ }^{2^{*}}$ \& Bo-An Li ${ }^{1,2^{*}}$ \\ ${ }^{1}$ State Key Laboratory of Cellular Stress Biology, Innovation Center for Cell Signaling Network and Engineering Research Center of Molecular \\ Diagnostics of the Ministry of Education, School of Life Sciences, Xiamen University, Xiamen 361005, China; \\ ${ }^{2}$ MOE Key Laboratory of Spectrochemical Analysis \& Instrumentation, Key Laboratory of Chemical Biology of Fujian Province, State Key \\ Laboratory of Physical Chemistry of Solid Surfaces, Collaborative Innovation Center of Chemistry for Energy Materials, Department of \\ Chemical Engineering, Department of Chemical Biology, College of Chemistry and Chemical Engineering, Xiamen University, \\ Xiamen 361005, China; \\ ${ }^{3}$ Department of Thoracic Surgery, The First Affiliated Hospital, Xiamen University, Xiamen 361005, China
}

Received August 17, 2021; accepted November 26, 2021; published online January 25, 2022

\begin{abstract}
Outbreaks of both influenza virus and the novel coronavirus SARS-CoV-2 are serious threats to human health and life. It is very important to establish a rapid, accurate test with large-scale detection potential to prevent the further spread of the epidemic. An optimized RPA-Cas12a-based platform combined with digital microfluidics (DMF), the RCD platform, was established to achieve the automated, rapid detection of influenza viruses and SARS-CoV-2. The probe in the RPA-Cas12a system was optimized to produce maximal fluorescence to increase the amplification signal. The reaction droplets in the platform were all at the microliter level and the detection could be accomplished within $30 \mathrm{~min}$ due to the effective mixing of droplets by digital microfluidic technology. The whole process from amplification to recognition is completed in the chip, which reduces the risk of aerosol contamination. One chip can contain multiple detection reaction areas, offering the potential for customized detection. The RCD platform demonstrated a high level of sensitivity, specificity (no false positives or negatives), speed ( $\leq 30 \mathrm{~min}$ ), automation and multiplexing. We also used the RCD platform to detect nucleic acids from influenza patients and COVID-19 patients. The results were consistent with the findings of qPCR. The RCD platform is a one-step, rapid, highly sensitive and specific method with the advantages of digital microfluidic technology, which circumvents the shortcomings of manual operation. The development of the RCD platform provides potential for the isothermal automatic detection of nucleic acids during epidemics.
\end{abstract}

nucleic acid detection, digital microfluidics, Cas12a, influenza virus, SARS-CoV-2

Citation: $\quad$ Sun Z, Lin KF, Zhao ZH, Wang Y, Hong XX, Guo JG, Ruan QY, Lu LY, Li X, Zhang R, Yang CY, Li BA. An automated nucleic acid detection platform using digital microfluidics with an optimized Cas12a system. Sci China Chem, 2022, 65: 630-640, https://doi.org/10.1007/s11426-021-1169-1

\section{Introduction}

The accurate detection of host pathogens is of great significance for the diagnosis of diseases, the evaluation of

$\uparrow$ These authors contributed equally to this work.

*Corresponding authors (email: bali@xmu.edu.cn; cyyang@xmu.edu.cn; raissarui@foxmail.com) treatment programs, the formulation of prevention and treatment measures, and the evaluation of drug resistance $[1,2]$. It is estimated that approximately 291,000 to 646,000 people worldwide die from respiratory diseases related to the seasonal influenza virus each year. Therefore, the effective detection of the influenza virus is a long-term task $[3,4]$. SARS-CoV-2 (previously named 2019-nCoV)-associated 
pneumonia, a newly found respiratory infectious disease, has swept the globe [5]. Tens of millions of people worldwide were confirmed to be infected as of 1 June, 2020. Due to limited sensitivity, traditional antigen/antibody-based methods are not suitable for the complete control of these epidemics [6]. Hence, nucleic acid detection has become an important means to accurately detect these pathogens.

Nucleic acid detection usually involves nucleic acid amplification steps. However, the traditional PCR technique requires stringent temperature control using a precise heating/cooling device to cycle through three steps, which is tedious and time-consuming. To circumvent the limitations of traditional PCR, many isothermal nucleic acid amplification methods have emerged, such as nucleic acid sequence-based amplification (NASBA) [7], strand displacement amplification (SDA) [8], loop-mediated isothermal amplification (LAMP) [9], and recombinase polymerase amplification (RPA) [10]. RPA and LAMP are currently the most promising isothermal amplification techniques. However, the sensitivity of LAMP is still slightly lower than that of qPCR, and the specificity of LAMP is inconsistent. Its specificity is comparable to that of qPCR for some pathogens and poor for others [11]. The sensitivity and specificity of RPA cannot be compared with those of qPCR method either [12]. Using a single isothermal amplification technique alone is not as effective as using qPCR. Thus, these methods need to be refined or combined with other techniques to increase performance.

In recent years, researchers have identified a unique group of Cas enzymes, including the Cas $12 \mathrm{a} / \mathrm{b}$, Cas13a and Cas14 proteins, that exhibit "collateral cleavage properties" when binding to the target nucleic acid sequence as an activator [13-16]. Detection platforms based on these proteins have been invented, such as "SHERLOCK", "DETECTR", "HOLMESv2", and "Cas14-DETECTR". SHERLOCK is a Cas13a-based molecular detection platform [17]. The target of the Cas13a protein is RNA, which causes the reaction to involve repeated transcription and reverse transcription $(\mathrm{RNA} \rightarrow \mathrm{DNA} \rightarrow \mathrm{RNA})$. DETECTR is a Cas12a-based method that achieves attomolar sensitivity for DNA detection [13]. HOLMESv2 is a Cas12b-assisted platform for nucleic acid detection [18]. Cas14-DETECTR is a Cas14based method that enables high-fidelity SNP genotyping. Cas14a protein can only recognize single-stranded DNA [19]. Among them, the "DETECTR" method, is the most widely developed because the Cas 12 a protein recognizes double-stranded DNA and the reaction temperature is the same as that required for RPA [17]. Since the outbreak of SARS-CoV-2, the field of Cas12a protein-based detection is advancing rapidly, for example, CRISPR-Cas12a-based lateral flow assay [20], a visual detection method based on Cas12a, AIOD-CRISPR [21], a one-step detection assay, deCOViD [22], a one-pot digitization-enhanced Cas12a-as- sisted detection assay, POC-CRISPR [23], a method of using point-of-care device for detection.

Here, we also propose a method that combines Cas12a detection with digital microfluidics (DMF) technology. DMF is a developing technology that uses tiny electrodes to manipulate fluids into discrete microdroplets and controls the reagent reaction on a substrate $[24,25]$. Based on the electrowetting-on-dielectric (EWOD) theory, the insulator becomes charged by applying an electric potential across the insulator on an array of electrodes. Individual droplets can be driven to move, merge, and separate by using electricity from electrodes on the substrate [26]. This process allows droplets to flow in the sealed chip, which prevents aerosol from evaporation. Moreover, it allows routine reactions to be performed with decreased time and reduced reagent consumption due to effective mixing [27]. We combined the RPA-Cas 12 a system with DMF to achieve the automated and sensitive detection of nucleic acids. This RPA-Cas12a-based platform combined with DMF is henceforth referred to as the RCD platform. We found a better probe to optimize the Cas12a system and designed the DMF chip suitable for nucleic acid detection. After that, the specificity and sensitivity of the RCD platform were verified. Finally, we used real samples from influenza patients and COVID-19 patients to verify the feasibility of the RCD method and found that the results of the RCD platform were consistent with those of real-time PCR. This finding indicates that the RCD method also has the prospect of practical application in nucleic acid detection.

\section{Materials and methods}

\subsection{Chemical materials}

All otherwise unspecified chemical reagents were obtained from Sangon Biotechnology Co., Ltd. (China). SU-8 2015 photoresist was purchased from Gersteltec Sarl (Pully, Switzerland). Teflon AF1600 was provided by DuPont (China). Dimethylsilicone oil was purchased from Aladdin (China). Pluronic F127 was provided by Sigma Aldrich (USA). All primers used in this study were synthesized and purified by Bioray Biotechnology Co., Ltd. (China). Probes and reporters were synthesized by Sangon Biotechnology Co., Ltd. (China). To compare the RCD platform with other established methods, we performed quantitative PCR (qPCR). Assays were performed using an Influenza Virus A/ B Real Time RT-PCR Kit (Liferiver, China).

\subsection{Chip design and manufacture}

The DMF chips were designed by AutoCAD software and consisted of two glass plates. The bottom glass plate was coated with 300 nm-thick chromium and patterned by ex- 
posure to ultraviolet light for $15 \mathrm{~s}$ through the principle of photolithography. The patterns were displayed on the chromium plate by using a developer for a positive photoresist. To form an electrode array, nitric acid chromium was used to corrode the chromium coating on the glass for $3 \mathrm{~min}$. The electrode array (Figure 1a) consisted of 40 actuation electrodes $(2.2 \mathrm{~mm} \times 2.2 \mathrm{~mm})$ and 8 reservoir electrodes $(5.4 \mathrm{~mm} \times 3.6 \mathrm{~mm})$. Then, we covered the plates with SU-8 2015 photoresist as a dielectric coating to create the droplet actuation phenomena and separate the electrodes. The top glass plates were coated with indium tin oxide (ITO). Both kinds of glass plates were eventually hydrophobized with Teflon AF 1600. Finally, the chips were heated to solidify the hydrophobic layer $\left(200^{\circ} \mathrm{C}, 20 \mathrm{~min}\right)$. A schematic diagram of the chip fabrication process is shown in Figure S1 (Supporting Information online). Three layers of double-sided tape (Scotch Brand, approximately $360 \mu \mathrm{m}$ ) were placed between the two glass plates to provide a space for droplet flow and prevent the two plates from sliding. The droplets were surrounded by dimethylsilicone oil to facilitate droplet manipulation and prevent evaporation. Dimethylsilicone oil was injected into the gap between the two plates with a syringe.

\subsection{RCD platform}

This automated system consisted of a DMF driving device $(40.8 \mathrm{~cm} \times 15.3 \mathrm{~cm} \times 5.6 \mathrm{~cm})$, DMF chips with readyprepared reagent, a heating plate and a fluorescence readout instrument (Figure S2). The DMF chip was placed on the chip holder, which was linked to the DMF driving generator via a pogo pin interface (48 pins). The method of purifying Cas12a protein was put in the Supporting Information online. The voltage of driving droplets is set to $120 \mathrm{Vrms}, 10 \mathrm{kHz}$. A heating plate set at $40{ }^{\circ} \mathrm{C}$ was placed under the chip to keep the temperature of droplets in the chip at about $37^{\circ} \mathrm{C}$. Reaction reagents were loaded on the reservoirs of the DMF chip in advance, and droplets were formed and driven to the intended sites. Finally, a fluorescence readout instrument (a microscope, DM2700 Leica, Nikon, Japan) was used to detect fluorescence and determine the presence of the pathogen through comparison with the fluorescence of the negative control. The wavelength range of the filter of the microscope was $490-530 \mathrm{~nm}$. The excitation light source for fluorescence detection was mercury lamp (EBQ 100-04,100W). The exposure time was $1 \mathrm{~s}$. The magnification was 40 times. A camera (Leica DFC7000T) was built into the microscope. The image analysis software we used was ImageJ $(1.48 \mathrm{v})$. The image depth in the software was set to 8 bit. Select the following three parameters: area, mean gray value, and integrated density. The brightness of the circular detection area in the image was converted into a value to represent the average intensity of fluorescence.

\subsection{Preparation of RNAs}

To determine the RNA sensitivity of the RCD platform, synthetic RNA (Table S1, Supporting Information online) was prepared. The T7 promoter sequence was added up-
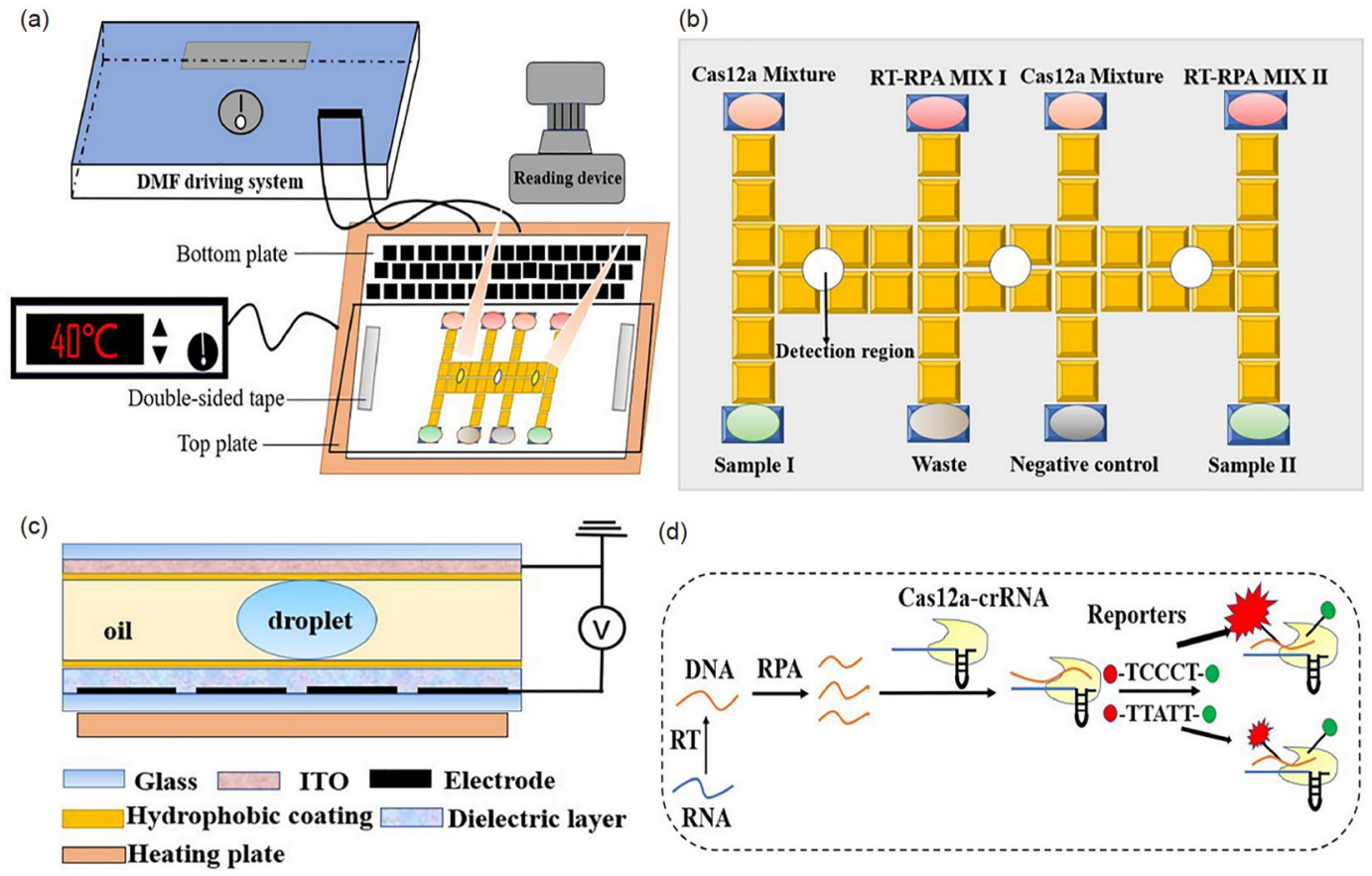

Figure 1 (a) Illustration of the RCD platform. (b) Plan view of the electrodes of the DMF chip and each reagent in the reservoir. (c) Side view of the DMF chip including a droplet. (d) Schematic illustration of the RPA-Cas12a-crRNA recognizing target and cleaving the reporter (color online). 
stream of the target sequence and then the whole sequence was inserted into $\mathrm{T}$ vector. The target RNA was produced using a HiScribeTM T7 Quick High Yield RNA Synthesis Kit (New England Biolabs). The preparation of crRNA is very similar to it. First, one part of the target sequence was chosen as the binding site of the crRNA. crRNA consists of a $5^{\prime}$ terminal fixed hairpin and a $3^{\prime}$ terminal single-stranded RNA. This single-stranded RNA was used to identify the target sequence. Generally, crRNA targeting sites were selected after RPA primer screening. The crRNA targeting position was between the two primers. The RNA design should contain minimal dimers and mismatches. The length of crRNA binding was approximately $20 \mathrm{nt}$. We used Primer 5 software to screen for sequences with relatively few mismatches and dimers. Then, the $\mathrm{T} 7$ promoter sequence was added upstream of the chosen sequence (Table S2). The crRNAs were produced by using a HiScribeTM T7 Quick High Yield RNA Synthesis Kit. The synthetic reactions were performed at $37^{\circ} \mathrm{C}$ overnight. Then, phenol-chloroform was used to extract the RNA and followed by ethanol precipitation. Finally, the RNAs were resuspended in $100 \mu \mathrm{L}$ of $\mathrm{ddH}_{2} \mathrm{O}$ (without RNase) and stored at $-80{ }^{\circ} \mathrm{C}$ for later use.

\subsection{Pseudovirus preparation and processing}

First, we prepared $120 \mu \mathrm{L}$ of HBSS with $1.5 \mu \mathrm{g}$ of pHR, $0.5 \mu \mathrm{g}$ of pVSVG, and $2 \mu \mathrm{g}$ of plasmids with the target nucleic acids inserted (listed in Table S3). Then, we mixed $81 \mu \mathrm{L}$ of HBSS with $9 \mu \mathrm{L}$ of $10 \times$ PEI and aspirated it approximately 20 times with a pipettor. Next, the second solution was put into the first solution and aspirated 20 times with a pipettor. The mixture was allowed to stand for $20 \mathrm{~min}$ and then we divided it into $293 \mathrm{~T}$ cells that were subjected to starvation in DMEM without FBS and antibiotics. After $8 \mathrm{~h}$ the medium was changed to DMEM containing 10\% FBS and $1 \%$ penicillin-streptomycin (ThermoFisher Scientific, USA). Forty-eight hours after changing the medium, the supernatant was filtered with $0.45 \mu \mathrm{m}$ filter (Minipore), and we used liquid nitrogen to freeze the supernatant. The viruses were stored at $-80^{\circ} \mathrm{C}$. The Magnetic Viral DNA/RNA Kit (TIANGEN) was used to isolate nucleic acids to test the sensitivity of our method in the laboratory. Finally, RNA was reversely transcribed to DNA by using a $5 \times$ All-In-One RT MasterMix Kit (Applied Biological Material, Canada).

\subsection{Clinical samples}

Nasopharyngeal swab samples were obtained from 33 patients with respiratory symptoms and 3 healthy people at The First Affiliated Hospital of Xiamen University from October to December in 2019. All samples were preserved in a $2 \mathrm{~mL}$ of Hank's buffer. One aliquot was retained by the hospital for clinical diagnosis, and another aliquot was sent to the la- boratory on ice within $2 \mathrm{~h}$. All the samples were stored at $-80{ }^{\circ} \mathrm{C}$ until RNA extraction. Nucleic acid purification from the nasopharyngeal swab samples was achieved with an Eastep ${ }^{\circledR}$ Super Total RNA Extraction Kit (Promega, Shanghai, China). Then, $4 \mu \mathrm{L}$ of the purified nucleic acids was injected into the pointed position of the chip and $6 \mu \mathrm{L}$ was used for qPCR. All samples were handled anonymously. Nucleic acid extraction from COVID-19 patient samples was completed in the laboratory of the First Affiliated Hospital of Xiamen University in 2020. Informed written consent was obtained from all participants prior to the research, and the samples were collected after informed consent and in agreement with the Institutional Review Board of The First Affiliated Hospital of Xiamen University. All detections were performed in an accredited laboratory. The results of this study did not influence the clinical discussion between patients and physicians.

\subsection{Recombinase polymerase amplification and Cas12a detection reagent}

The ZC-RAA ${ }^{\circledR}$ Basic and ZC-RAA ${ }^{\circledR}$ Basic RT kits were purchased from ZC Bio-Sci \& Tech Co., Ltd. (China). It should be noted that, recombinase-aided amplification (RAA) and RPA are both recombinant enzyme-based amplification methods. Both of the principles and the amplification effect are similar [28]. Reaction temperatures are both about $37^{\circ} \mathrm{C}$. The amplification reagent is configured according to the instructions, and the concentration of the primers is $0.4 \mu \mathrm{M}$. The Cas $12 \mathrm{a}$ reaction mix used in RCD platform consisted of purified Cas 12 a protein $(0.16 \mu \mathrm{g} / \mu \mathrm{L})$, crRNA $(0.02 \mu \mathrm{g} / \mu \mathrm{L})$, reporters with fluorescence quenching groups $(0.8 \mu \mathrm{M})$ (Table $\mathrm{S} 3)$, reaction buffer $(100 \mathrm{mM} \mathrm{NaCl}$, 50 Tris, $10 \mathrm{mM} \mathrm{MgCl}_{2}, 1 \mathrm{mM}$ DTT, pH 7.9) and $0.008 \%$ Pluronic F127 solution. We used Primer 5 software to design primers for RPA/RT-RPA with a primer size of 31-34 nt and a primer melting temperature of $65^{\circ} \mathrm{C}$. In the experiment of screening probes, we used a $50 \mu \mathrm{L}$ system. The system includes Cas12a protein $(0.16 \mu \mathrm{g} / \mu \mathrm{L})$, crRNA $(0.02 \mu \mathrm{g} / \mu \mathrm{L})$, reporters $(0.8 \mu \mathrm{M})$, target plasmid in Table $\mathrm{S} 1(0.1 \mu \mathrm{g} / \mu \mathrm{L})$ and reaction buffer $\left(100 \mathrm{mM} \mathrm{NaCl}, 50\right.$ Tris, $10 \mathrm{mM} \mathrm{MgCl}_{2}$, $1 \mathrm{mM}$ DTT, pH 7.9). The reaction was carried out in 96 well plates. After reaction at $37^{\circ} \mathrm{C}$ for $1 \mathrm{~h}$, the final fluorescence value was measured in an enzyme labeling instrument (Tecan Spark, Switzerland).

\subsection{Data analysis}

Statistical analysis and the drawing of the statistical chart were performed with Graphpad Prism software (8.0.2v). The Student's t-test was used to compare the means of two independent groups to each other. $P<0.05$ was considered that there was a significant difference between the two groups. 


\section{Results and discussion}

\subsection{Working principle of the RCD platform}

Figure 1 illustrates the working principle of the RCD platform for automated multiple nucleic acid detection. The overall diagram of the RCD platform is displayed in Figure 1a. The whole detection process is carried out on the chip and read by the instrument after the reaction. Since the aperture of the detection area on the chip is very small, we used a microscope with a built-in camera function to collect the image. The RCD procedure was conducted as follows: $2 \mu \mathrm{L}$ of isolated nucleic acids and $2 \mu \mathrm{L}$ of the RT-RPA mix were blended under the direction of a computer program, and the mixture flowed along the electrodes for $10 \mathrm{~min}$. Next, the droplet was split into two small identical droplets, from which one small droplet was moved to the waste reservoir, while the other droplet was incubated with $2 \mu \mathrm{L}$ of Cas12acrRNA-probe mix for $20 \mathrm{~min}$. Driving the flow of larger droplets requires a higher voltage, which increases the risk of electrodes failure. Thus, we removed $2 \mu \mathrm{L}$ volume from the droplet after the RT-RPA reaction to keep the subsequent reaction volume at $4 \mu \mathrm{L}$ (Figure S3). To perform multiple reactions, multiple regions could be designed on the chip. In our experiments, three detection regions were arranged (circular areas on the chip), including one for the control and two for the samples (Figure 1b). The side view of the chip is shown in Figure 1c. The bottom plate is divided into four layers from bottom to top: the glass layer, the electrode, the dielectric layer, and the hydrophobic layer. The top plate is divided into three layers from top to bottom: the glass layer, the indium tin oxide (ITO) layer, and the hydrophobic layer. The droplets react between the top and bottom plates of the chip. The droplets are surrounded by silicone oil to prevent liquid evaporation and aerosol pollution. Figure 1d illustrates the principle of detection. Once the Cas12a-crRNA complexes match the target sequence amplified by RPA, cleavage of the reporter flanked by the fluorescence (5-FAM) group and quenching (BHQ1) group will occur. Specific details are given in the Materials and Methods.

\subsection{Optimization of the fluorescent probe in Cas12a system}

In the published CRISPR-related detection methods, the length of the probe is in the range of 2-12 nucleotides. The probe length of Cas13a protein is 2-7 nucleotides [29], the probe base length of the Cas $12 \mathrm{~b}$ and Cas $14 \mathrm{a}$ proteins is 12 nucleotides [18,19]. All the published probes for Cas12a protein are 5 nucleotides $[13,30]$. Therefore, we designed probes (poly-T) with different lengths and explored the preference of the Cas $12 \mathrm{a}$ protein for probe lengths in the range of 2-12 nucleotides. We found that the Cas12a protein was not sensitive to probes that were too short $(<4$ nucleo- tides). The signal-to-background ratio increased with increasing probe length but reached a peak when the length was 5. Subsequently, the probe length no longer affected the signal-to-background ratio (Figure 2a). Then, we explored whether the Cas12a protein has a preference for nucleotide sequences similar to the Cas13a protein. We designed probes with different nucleotide sequences and found that the nucleotide sequence in the probe could also affect the fluorescence. In Figure $2 b$, we found that when guanines $(G)$ were connected, the fluorescence produced by the probe became much lower. This is very similar to the properties of TaqMen probes. "TCCCT" was observed to exhibit the strongest signal-to-background ratio compared with those of the other sequences and was twice that of "TTATT" chosen by the SHERLOCK and DETECTER methods. No study has yet revealed the structural principle of the collateral cleavage property of the CRISPR protein. We speculate that the CRISPR protein may be structurally similar to the Taq enzyme, but further structural research is needed. Finally, we chose "FAM-TCCCT-BHQ1" as a probe.

\subsection{Working performance of the DMF chip}

The stability of the reagent droplet volume is an important factor affecting the detection performance [31]. We evaluated the uniformity of the liquid volume distribution of the chip. Since the amplification reagent contained a large amount of protein, the viscosity of the reagent was relatively high. We added Pluronic F127 to all the reagents to prevent the droplets from adhering to the chip surface [32]. First, the volume of droplets dispensed from one reservoir was investigated. The gap between the top and bottom plates is fixed, so the droplet area determines the droplet volume. We used a camera to record the droplet areas and calculated them by using ImageJ (Figure S4). A video of dispensing a droplet was captured in the Supporting Information online. Twenty droplets were dispensed from one reservoir on the same chip and the coefficient of variation (CV) of their areas was $4.2 \%$ (Figure 3a). Then, the liquid distribution of the other reservoirs was tested and the CV was $3.8 \%$ (Figure $3 b$ ). The coefficient of variation is the ratio of the standard deviation of the data to the mean. Generally, the smaller the coefficient of variation is, the smaller the degree of data dispersion is. The low dispersion coefficient of the dispersed droplet volume indicates that the liquid volume distribution of the chip is very stable. The stability of the dispersed droplets in the chip ensures the consistency of each reaction.

\subsection{Sensitivity and specificity of the RCD platform}

To determine the sensitivity of the RCD platform, a $399 \mathrm{bp}$ nucleic acid sequence (Table S1) was inserted into the $\mathrm{T}$ vector as the DNA target plasmid. Gradient dilutions of the 

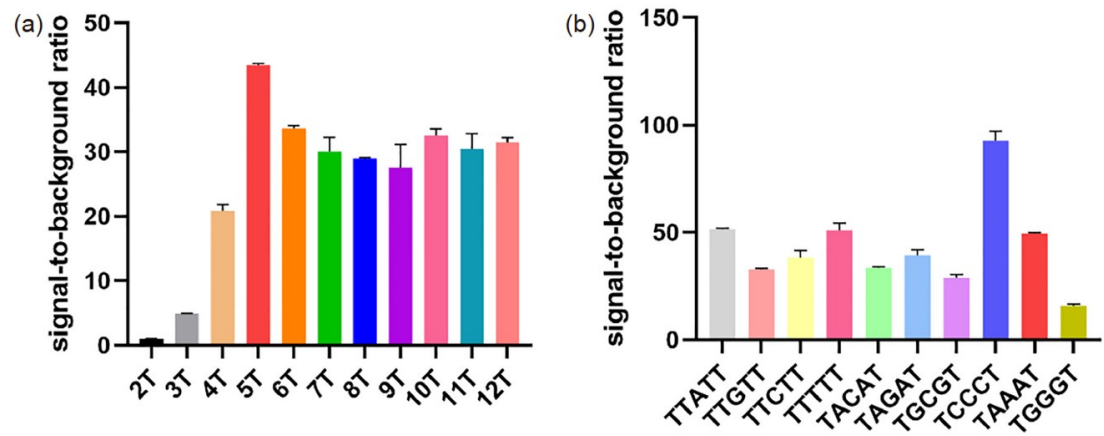

Figure 2 Screening of the fluorescence probes in the RCD platform. (a) The length of the probe could influence the signal-to-background ratio. (b) Nucleotide sequences could also influence the signal-to-background ratio. Each bar represents the average value of three technical replicates (color online).
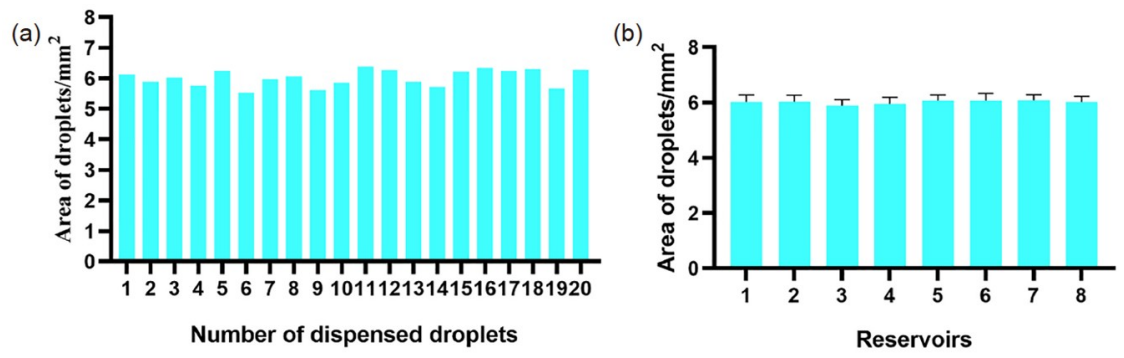

Figure 3 The performance of chip dispersed droplets. (a) The area of 20 droplets dispensed from one reservoir with a CV (\%) of $4.2 \%$. (b) The area of 20 droplets dispensed from different reservoirs with a CV (\%) of 3.8\%. Each bar represents the average value of three technical replicates (color online).

plasmid were prepared, and the dilutions were quantified by using the ddPCR technique (Figure S5). When we tested the RCD platform by using the target plasmid, we achieved single-molecule sensitivity, and the coefficient of variation (CV) of fluorescence was $6.61 \%$ (Figure $4 \mathrm{a}$ ). To determine the RNA sensitivity of the RCD platform, we prepared synthetic RNA. We used commercial RT-RPA reagents, and reverse transcription and amplification were carried out together. We diluted the RNA in a tenfold gradient and found that the fluorescence decreased significantly at 10 copies (Figure $4 b$ ). We narrowed the detection range again to determine the LOD value. The fluorescence is approximately linear between 10 and 60 copies. Three negative controls of this experiment were placed in Figure S6. In general, the LOD value is equal to three times the standard deviation of the negative control divided by the slope of the straight line [33]. After calculation, the LOD value of RNA detected by the RCD platform was approximately 5.2 copies (Figure $4 \mathrm{c}$ ), and the fluorescence at the LOD was approximately 1.5 times that of negative fluorescence. In actual detection, improperly preserved samples and extraction loss will reduce the amount of extracted nucleic acid, so high sensitivity is the basic guarantee for accurate detection of the RCD platform.

The light source of the microscope is a mercury lamp. With the increase of service time, the brightness of the mercury lamp decreases gradually. Therefore, the brightness of dif- ferent groups would be slightly different and we did negative control in each group of experiments. Some bright spots were observed in Figure 4a, which can also be found in the following figures. We deduced that this phenomenon was caused by the high concentration of polyethylene glycol (PEG) in the RPA reagents. With increasing reaction time, the nucleic acid was expanded exponentially, and PEG could enrich the nucleic acid-Cas12a-crRNA complex and accumulate it into a luminescent precipitate. However, this phenomenon could not be observed in the control or negative samples. Thus, we concluded that the precipitation did not affect the test results. In the future, we will continue to optimize the amplification system in the chip to reduce its aggregation and sedimentation.

We identified two groups of viruses to verify the specificity of the RCD platform. The principle of specificity detection is to detect pathogens with homology or similar clinical symptoms. The first group of viruses was influenza virus A (A/California/04/2009/H1N1), influenza virus B (B/ Florida/04/2006/Yamagata) and respiratory syncytial virus (RSV). The matrix protein (MP) gene is fairly conserved among all influenza virus subtypes [34]. We designed degenerate primers to circumvent the problem that a few mutations do exist in the MP region. The screened primers can be used to cover common influenza A viruses, such as H1N1, H3N2, H5N1 and H7N9 [35-38]. Moreover, we designed primers for the influenza B virus by the same method (Fig- 

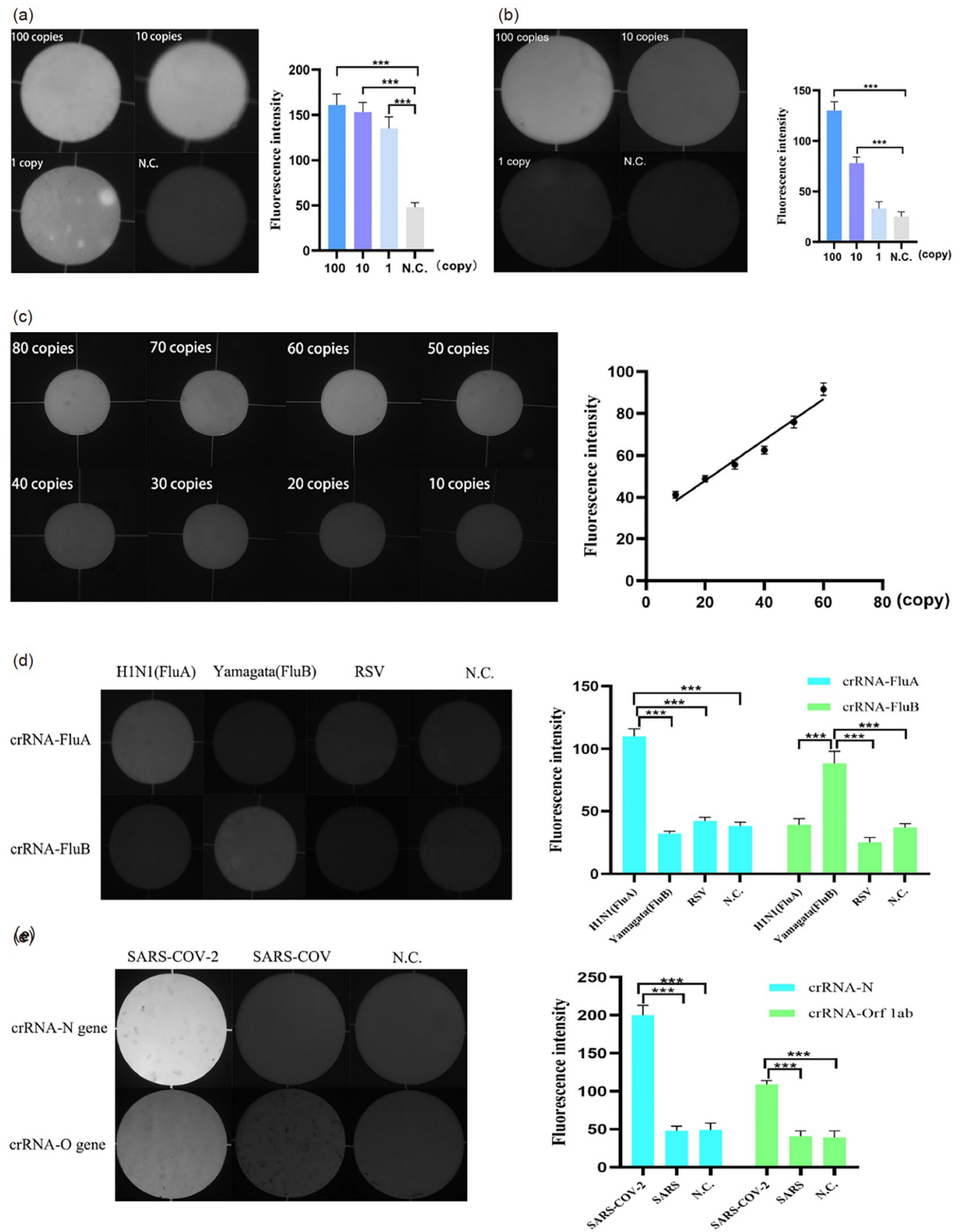

Figure 4 The sensitivity and specificity of the RCD platform. (a) Fluorescence image of target DNA detection and quantification of the fluorescence density. (b) Fluorescence image of target RNA detection and quantification of the fluorescence density. (c) Fluorescence image of lower concentrations of RNA detection and quantification of the fluorescence density. (d) Fluorescence image of cross-reaction detection among influenza A virus, influenza B viruses and respiratory syncytial virus, followed by quantification of the fluorescence densities. (e) Fluorescence image of cross-reaction detection between SARS-CoV-2 and SARS and quantification of the fluorescence densities. Each bar represents the average value of three technical replicates. ${ }^{* * *} P<0.001$ (color online).

ures S7, S8). The second group of viruses was SARS-CoV-2 and SARS virus. At present, the most common nucleic acid detection method to detect SARS-CoV-2 is to test speciesspecific regions of the SARS-CoV-2 genome, such as the nucleoprotein $(\mathrm{N})$ gene, envelope $(\mathrm{E})$ genes, and open reading frame $1 \mathrm{ab}$ (Orf 1ab) gene. To improve the accuracy of detection, we carried out double gene detection of the SARS-CoV-2, N gene and Orf $1 \mathrm{ab}$ gene. We prepared pseudoviruses containing the $\mathrm{N}$ gene and Orf $1 \mathrm{ab}$ gene of SARS-CoV-2 and SARS viruses. crRNAs of each gene were designed and are listed in Table S3. Viruses of each group were diluted in a gradient and we used the same concentra- 
tions as input for the detection of each virus. The results showed no cross-reaction (Figure 4d, e). In conclusion, these results suggest a convincing sensitivity and specificity for the RCD platform and indicate that the RCD platform is suitable for pathogen detection.

\subsection{Feasibility of the RCD platform for use on real clinical samples}

Real clinical samples of influenza patients were detected to verify the feasibility of the RCD platform. We collected 33 swab samples from patients with respiratory symptoms at The First Affiliated Hospital of Xiamen University from October to December in 2019. These samples had been diagnosed by antigen-antibody detection, and 2 cases were negative. We also collected swab samples from three healthy people. Informed written consent was obtained from all participants prior to the research and the samples were collected after informed consent and in agreement with the Institutional Review Board of The First Affiliated Hospital of Xiamen University. Then these 36 samples were tested in the laboratory by the RCD platform (Figure 5) and real-time PCR method (Figure S9). In the RCD platform, the difference between negative and positive in these samples can be distinguished by the naked eye. ImageJ software was also used to quantify the brightness of the results. We calculated the ratio of the fluorescence of all samples to that of negative control (no input).

We defined the cut-off value of the positive result as 1.5 times of the tested sample versus the negative control. The fluorescence ratio of 31 samples identified as positive was greater than 1.5, and the fluorescence ratio of the remaining 5 samples (including 3 samples from healthy people) was approximately 1 . These 36 samples were also tested by realtime PCR, and we compared the fluorescence of real-time PCR with that of the RCD platform. For negative results in the RCD platform, the real-time PCR results did not show a peak. For these 36 samples, the RCD platform achieved a $100 \%$ detection rate.

Figure 5 also shows that the fluorescence of the influenza $B$ virus sample is somewhat stronger than that of the influenza A virus sample. We think this is because we used degenerate primers in the system. Because influenza A virus contains a large number of mutations, we designed primers (Figure S8) for the most common genotypes (H1N1, H3N2, H5N1 and H7N9). The primer concentration of a certain variety, such as $\mathrm{H} 1 \mathrm{~N} 1$, was therefore relatively low. Accordingly, under the same reaction conditions, the amplification efficiency of the influenza A virus was not as high as that of the influenza B virus. Nevertheless, we distinguished the patients from the healthy person, and the typing was very accurate. Improving reaction efficiency is a major advantage of DMF technology. In addition, we used a probe (TCCCT) with higher fluorescence intensity. These two advantages made up for the relative shortage of primers. Therefore, the RCD platform achieved large-area coverage of the detection of variant viruses, which may help the medical staff determine the treatment plan and prevention and control measures correctly. This proves that the platform is also suitable for viruses with mutations.

Fourteen swab samples from COVID-19 patients and 2 swab samples from healthy people were collected from the

\begin{tabular}{|c|c|c|c|c|c|}
\hline$A$ & A & $A$ & A & $A$ & $A$ \\
\hline$P$ & $P$ & $P$ & $P$ & $P$ & $P$ \\
\hline 3.7 & 3.4 & 2.4 & 2.5 & 2.2 & 2.7 \\
\hline A & A & $A$ & A & A & A \\
\hline$P$ & $\mathrm{P}$ & $P$ & $P$ & $P$ & $P$ \\
\hline 2.4 & 3.1 & 2.6 & 3.0 & 3.1 & 3.2 \\
\hline A & A & A & A & A & A \\
\hline$P$ & $P$ & $P$ & $P$ & $P$ & $P$ \\
\hline 2.9 & 2.2 & 2.3 & 3.0 & 2.3 & 2.5 \\
\hline$A$ & $A$ & $A$ & $A$ & $A$ & B \\
\hline$P$ & $P$ & $P$ & $P$ & $P$ & $P$ \\
\hline 2.8 & 2.7 & 3.0 & 3.5 & 3.8 & 6.2 \\
\hline B & B & B & B & B & B \\
\hline$P$ & $P$ & $\mathrm{P}$ & $P$ & $\mathrm{P}$ & $\mathrm{P}$ \\
\hline 5.1 & 4.5 & 4.2 & 4.4 & 4.9 & 3.7 \\
\hline B & $\mathrm{N}$ & $\mathrm{N}$ & $\mathrm{H}$ & $\mathrm{H}$ & $\mathrm{H}$ \\
\hline$P$ & $\mathrm{~N}$ & $\mathrm{~N}$ & $\mathrm{~N}$ & $\mathrm{~N}$ & $\mathrm{~N}$ \\
\hline 4.3 & 1.3 & 0.9 & 1.2 & 1.4 & 0.9 \\
\hline
\end{tabular}
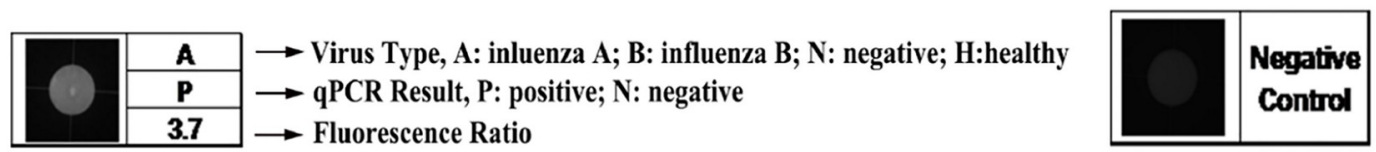

Figure 5 Influenza virus detection in 36 swab samples with the RCD platform. 
hospital and nucleic acids were extracted in the hospital laboratory. Double genes among these nucleic acids were detected by the RCD platform. The cut-off value is still defined as 1.5 times the tested sample versus the negative control. All the samples were successfully detected and the results coincided with the facts (Figure 6). Because these 14 samples had been confirmed negative and positive in the hospital, and the amount of nucleic acid extracted was very small, we used only the RCD platform to test the samples. Samples were collected at different times, so we tested the negative control for each sample. There were no false-positive results in the detection. This result also shows that the RCD platform is a reliable potential platform for infectious disease detection. Because the chip is made by our laboratory, manual degumming can easily leave residues in the pattern-making process. Residual debris can be observed under the microscope in Figure 6. Because the debris is located under the dielectric layer and the amount of debris is small, it does not affect the flow of the droplets. This is also confirmed in Figure 3.

By detecting influenza samples, we compared the RCD platform with qPCR and the antigen-antibody method in terms of reaction time, sensitivity, cost and reaction temperature (Table S4). The reaction time of qPCR is greater than that of the antigen-antibody method and the RCD platform. The antigen-antibody method and the RCD platform can both be used at a constant temperature. qPCR requires repeated temperature changes, so it is time-consuming $(>1 \mathrm{~h})$ and requires complex instruments. The time of antibody production in the human body varies from person to person, and false-negative results often occur in the antigenantibody method. The sensitivity of the antigen-antibody method is approximately 65\% [39]. Thus, the antigenantibody method is not as sensitive as qPCR or the RCD platform. The reagent cost of qPCR is not high, but bulky and complex instruments were unfavorable for a rapid diagnostic field test. The RCD platform requires only a few microliters of reagents, and the reagent cost is even lower than that of qPCR. The cost of the artificial chip in the lab is approximately 35 RMB per chip (Table S4). A simple blue lightemitting detection device can be customized and the driving device of the chip is less technical than a qPCR instrument
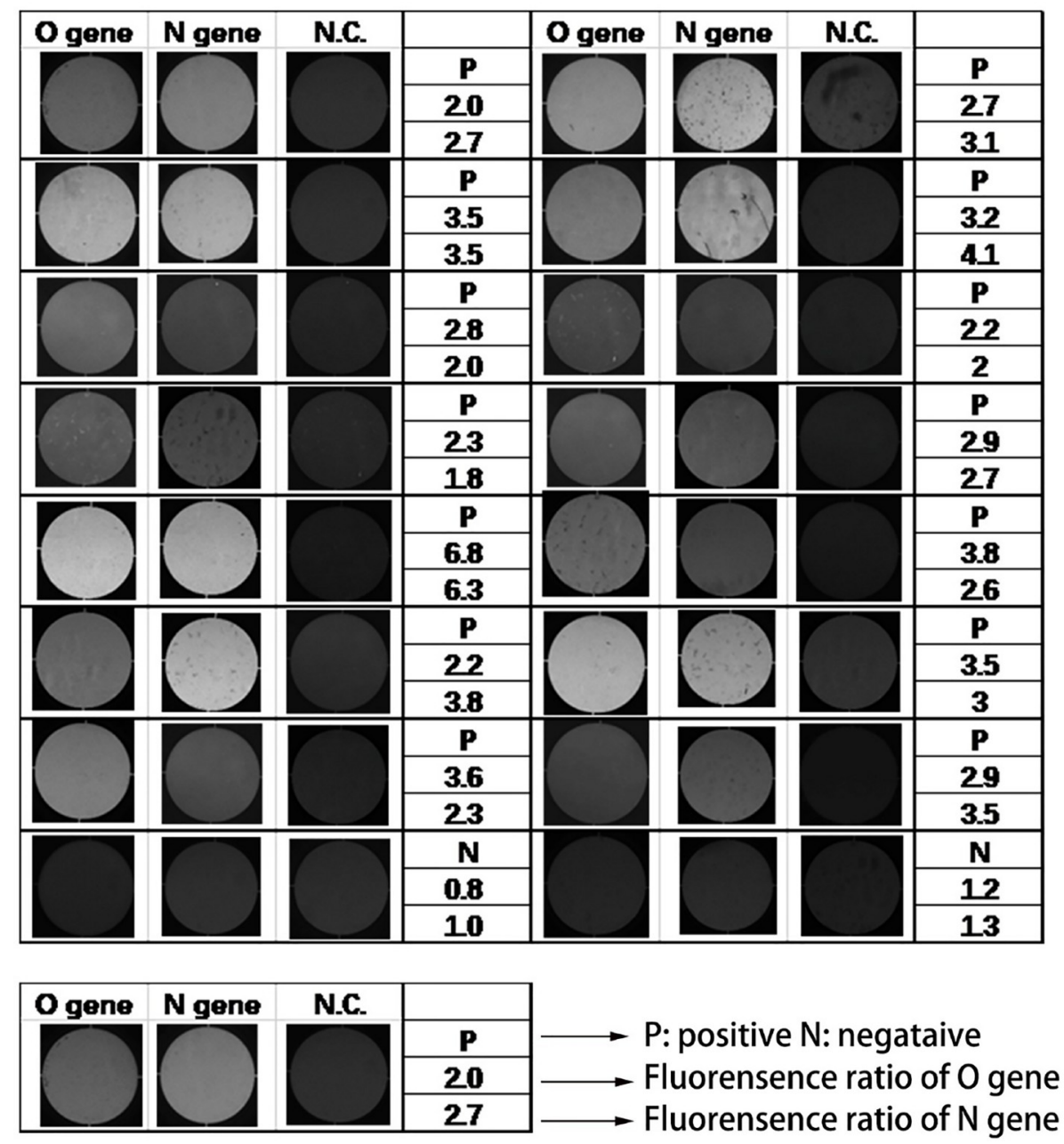

Figure 6 SARS-CoV-2 detection of double genes in 16 nucleic acid samples with the RCD platform. 
and easy to carry, which is more conducive to rapid detection in the future. Overall, the RCD platform combines the advantages of qPCR and antigen-antibody method, with high sensitivity, fast reaction time and constant temperature. The cost of the RCD platform is expected to decline significantly after large-scale industrial production. It is hoped in the future that the marginal cost of the RCD platform per reaction is equal to that of qPCR.

\section{Conclusions}

In conclusion, the RCD platform was successfully developed for the detection of pathogens, especially RNA viruses. The platform has proven to be highly sensitive and specific under laboratory conditions. The amplification by RPA and the optimization of Cas12a-crRNA ensured the sensitivity of the system. The new fluorescence reporter (TCCCT) further optimized the platform. The dispersed droplets of the chip are uniform and stable. The reliable detection of virus in 52 real clinical samples has validated its ability to be used in community hospitals, clinics and laboratories for pathogen diagnosis. Compared with other methods, such as the antigen-antibody method and qPCR, the RCD platform offers advantages in terms of high sensitivity, low time cost, and the ability to perform the reaction at a constant temperature. With the DMF technique, the RCD platform presents the following advantages. First, the automation of DMF greatly simplifies the operation and reduces the labor required. Second, the chip integrates multiple electrodes, which can be used for multichannel detection in the future. Third, DMF prevents the risk of nucleic acid contamination caused by lid opening. Furthermore, the RCD platform can be highly applicable for various tasks, including immunoassay applications [31], cell-based applications [40], and SNP genotyping. Our RCD platform is still in the early stage, and in the future, we will improve the microfluidic throughput and chip properties. We will develop amplification systems that do not produce precipitation. Moreover, we will increase the number of detection areas on the chip. Instruments and software for controlling multiple chips at one time will be developed.

\footnotetext{
Acknowledgements This work was supported by the Science and Technology Program of Fujian Province (2018Y4013 to B.-A.L.), the Science and Technology Project of Xiamen Science and Technology Bureau (3502Z20193023 to B.-A.L.), the Health-Education Joint Research Project of Fujian Province (2019-WJ-34 to B.-A.L. and Z.-M.Z), the COVID-19 Emergency Research Project of Xiamen Science and Technology Bureau (3502Z2020YJ21 to BioDetect (Xiamen) Biotechnology Co., Ltd.), the COVID-19 Emergency Research Project of Xiamen University (X2106103 to B.-A.L.), the National Natural Science Foundation of China (U1705284, 81972458, and 81772958 to B.-A.L.) and Project 111 sponsored by the State Bureau of Foreign Experts and Ministry of Education (B06016).
}

Conflict of interest The authors declare no conflict of interest.
Supporting information The supporting information is available online at http://chem.scichina.com and http://link.springer.com/journal/11426. The supporting materials are published as submitted, without typesetting or editing. The responsibility for scientific accuracy and content remains entirely with the authors.

1 Waggoner JJ, Pinsky BA. J Clin Microbiol, 2016, 54: 860-867

2 Falsey AR, Murata Y, Walsh EE. Arch Intern Med, 2007, 167: 354

3 Bi Y, Chen Q, Wang Q, Chen J, Jin T, Wong G, Quan C, Liu J, Wu J, Yin R, Zhao L, Li M, Ding Z, Zou R, Xu W, Li H, Wang H, Tian K, Fu G, Huang Y, Shestopalov A, Li S, Xu B, Yu H, Luo T, Lu L, Xu X, Luo Y, Liu Y, Shi W, Liu D, Gao GF. Cell Host Microbe, 2016, 20: $810-821$

4 Taubenberger JK, Reid AH, Krafft AE, Bijwaard KE, Fanning TG. Science, 1997, 275: 1793-1796

5 V'kovski P, Kratzel A, Steiner S, Stalder H, Thiel V. Nat Rev Microbiol, 2021, 19: 155-170

6 Day MJ. Top Companion Anim Med, 2015, 30: 128-131

7 Compton J. Nature, 1991, 350: 91-92

8 Walker GT, Fraiser MS, Schram JL, Little MC, Nadeau JG, Malinowski DP. Nucl Acids Res, 1992, 20: 1691-1696

9 Notomi T, Okayama H, Masubuchi H, Yonekawa T, Watanabe K, Amino N, Hase T. Nucl Acids Res, 2000, 28: 63e-63

10 Piepenburg O, Williams CH, Stemple DL, Armes NA. PLoS Biol, 2006, 4: e204

11 Aebischer A, Wernike K, Hoffmann B, Beer M. J Clin Microbiol, 2014, 52: 1883-1892

12 Song J, Liu C, Mauk MG, Rankin SC, Lok JB, Greenberg RM, Bau HH. Clin Chem, 2017, 63: 714-722

13 Chen JS, Ma E, Harrington LB, Da Costa M, Tian X, Palefsky JM, Doudna JA. Science, 2018, 360: 436-439

14 Teng F, Guo L, Cui T, Wang XG, Xu K, Gao Q, Zhou Q, Li W. Genome Biol, 2019, 20: 132

15 Abudayyeh OO, Gootenberg JS, Konermann S, Joung J, Slaymaker IM, Cox DBT, Shmakov S, Makarova KS, Semenova E, Minakhin L, Severinov K, Regev A, Lander ES, Koonin EV, Zhang F. Science, 2016, 353

16 Aquino-Jarquin G. Nanomed-Nanotechnol, 2019, 18: 428-431

17 Gootenberg JS, Abudayyeh OO, Lee JW, Essletzbichler P, Dy AJ, Joung J, Verdine V, Donghia N, Daringer NM, Freije CA, Myhrvold C, Bhattacharyya RP, Livny J, Regev A, Koonin EV, Hung DT, Sabeti PC, Collins JJ, Zhang F. Science, 2017, 356: 438-442

18 Li L, Li S, Wu N, Wu J, Wang G, Zhao G, Wang J. ACS Synth Biol, 2019, 8: 2228-2237

19 Harrington LB, Burstein D, Chen JS, Paez-Espino D, Ma E, Witte IP, Cofsky JC, Kyrpides NC, Banfield JF, Doudna JA. Science, 2018, 362: 839-842

20 Broughton JP, Deng X, Yu G, Fasching CL, Servellita V, Singh J, Miao X, Streithorst JA, Granados A, Sotomayor-Gonzalez A, Zorn K, Gopez A, Hsu E, Gu W, Miller S, Pan CY, Guevara H, Wadford DA, Chen JS, Chiu CY. Nat Biotechnol, 2020, 38: 870-874

21 Ding X, Yin K, Li Z, Lalla RV, Ballesteros E, Sfeir MM, Liu C. Nat Commun, 2020, 11: 4711

22 Park JS, Hsieh K, Chen L, Kaushik A, Trick AY, Wang TH. Adv Sci, 2021, 8: 2003564

23 Chen FE, Lee PW, Trick AY, Park JS, Chen L, Shah K, Mostafa H, Carroll KC, Hsieh K, Wang TH. Biosens Bioelectron, 2021, 190: 113390

24 Cho SK, Moon HJ, Kim CJ. J Microelectromech Syst, 2003, 12: 70-80

25 Squires TM, Quake SR. Rev Mod Phys, 2005, 77: 977-1026

26 Choi K, Ng AHC, Fobel R, Wheeler AR. Annu Rev Anal Chem, 2012, 5: $413-440$

27 Kumar PT, Toffalini F, Witters D, Vermeir S, Rolland F, Hertog MLATM, Nicolaï BM, Puers R, Geeraerd A, Lammertyn J. Sens Actuat B-Chem, 2014, 199: 479-487

28 Fan X, Li L, Zhao Y, Liu Y, Liu C, Wang Q, Dong Y, Wang S, Chi T, 
Song F, Sun C, Wang Y, Ha D, Zhao Y, Bao J, Wu X, Wang Z. Front Microbiol, 2020, 11: 1696

29 Gootenberg JS, Abudayyeh OO, Kellner MJ, Joung J, Collins JJ, Zhang F. Science, 2018, 360: 439-444

30 Li SY, Cheng QX, Liu JK, Nie XQ, Zhao GP, Wang J. Cell Res, 2018, 28: 491-493

31 Wang Y, Ruan Q, Lei ZC, Lin SC, Zhu Z, Zhou L, Yang C. Anal Chem, 2018, 90: 5224-5231

32 Au SH, Kumar P, Wheeler AR. Langmuir, 2011, 27: 8586-8594

33 Zhu Z, Guan Z, Liu D, Jia S, Li J, Lei Z, Lin S, Ji T, Tian Z, Yang CJ. Angew Chem Int Ed, 2015, 54: 10448-10453

34 Zheng M, Luo J, Chen Z. Infection, 2014, 42: 251-262

35 Taubenberger JK, Kash JC. Cell Host Microbe, 2010, 7: 440-451

36 Webster RG, Peiris M, Chen H, Guan Y. Emerg Infect Dis, 2006, 12:
3-8

37 Gao R, Cao B, Hu Y, Feng Z, Wang D, Hu W, Chen J, Jie Z, Qiu H, Xu K, Xu X, Lu H, Zhu W, Gao Z, Xiang N, Shen Y, He Z, Gu Y, Zhang Z, Yang Y, Zhao X, Zhou L, Li X, Zou S, Zhang Y, Li X, Yang L, Guo J, Dong J, Li Q, Dong L, Zhu Y, Bai T, Wang S, Hao P, Yang W, Zhang Y, Han J, Yu H, Li D, Gao GF, Wu G, Wang Y, Yuan Z, Shu Y. N Engl J Med, 2013, 368: 1888-1897

38 Obenauer JC, Denson J, Mehta PK, Su X, Mukatira S, Finkelstein DB, Xu X, Wang J, Ma J, Fan Y, Rakestraw KM, Webster RG, Hoffmann E, Krauss S, Zheng J, Zhang Z, Naeve CW. Science, 2006, 311: 15761580

39 Ghebremedhin B, Engelmann I, König W, König B. J Med Microbiol, 2009, 58: 365-370

40 Aijian AP, Garrell RL. J Lab Autom, 2015, 20: 283-295 